\title{
Nonexistence of Nontrivial Solutions with Decay Order for a Biharmonic P-Laplacian Equation and System
}

\author{
Jeng-Eng Lin \\ Department of Mathematics and Statistics, Georgetown University, Washington DC, USA \\ Email: JengEng.Lin@georgetown.edu
}

How to cite this paper: Lin, J.-E. (2017) Nonexistence of Nontrivial Solutions with Decay Order for a Biharmonic P-Laplacian Equation and System. Applied Mathematics, 8, 920-928.

https://doi.org/10.4236/am.2017.87072

Received: May 12, 2017

Accepted: July 9, 2017

Published: July 12, 2017

Copyright $\odot 2017$ by author and Scientific Research Publishing Inc. This work is licensed under the Creative Commons Attribution International License (CC BY 4.0).

http://creativecommons.org/licenses/by/4.0/ (c) (i)

\begin{abstract}
We use the Morawetz multiplier to show that there are no nontrivial solutions of certain decay order for a biharmonic equation with a p-Laplacian term and a system of coupled biharmonic equations with p-Laplacian terms in the entire Euclidean space.
\end{abstract}

\section{Keywords}

Morawetz Multiplier, Biharmonic, P-Laplacian, Nonexistence

\section{Introduction}

Recently, there has been an active research on the biharmonic equation with a p-Laplacian term

$$
\Delta^{2} u+\alpha \nabla \cdot\left(|\nabla u|^{p-2} \nabla u\right)+\beta \cdot u+f(\boldsymbol{x}, u)=0
$$

as well as the evolutionary biharmonic equations with a p-Laplacian term

$$
\partial u / \partial t=\Delta^{2} u+\alpha \nabla \cdot\left(|\nabla u|^{p-2} \nabla u\right)+\beta \cdot u+f(x, u)
$$

and

$$
\partial^{2} u / \partial t^{2}=\Delta^{2} u+\alpha \nabla \cdot\left(|\nabla u|^{p-2} \nabla u\right)+\beta \cdot u+f(x, u)
$$

where $\boldsymbol{x} \in \boldsymbol{R}^{n}, n \geq 2, p>1, \alpha$ and $\beta$ are real constants.

Equation (1.1) is the stationary state of the Equation (1.2) while the traveling wave solution for (1.3) satisfies an equation of the form (1.1) as was shown in Strauss [1]. For the analysis and applications of (1.1), (1.2), and (1.3), see, for example, [2]-[42]. In this article, we shall use the Morawetz multiplier [43] [44] [45] to show that there are no nontrivial solutions of certain decay order for (1.1) 
and a system of coupled biharmonic equations with p-Laplacian terms,

$$
\begin{gathered}
\Delta^{2} u+\alpha \nabla \cdot\left(|\nabla u|^{p-2} \nabla u\right)+\beta \cdot u+f(\boldsymbol{x}, u)+\left[a(\boldsymbol{x}) u^{2}+b(\boldsymbol{x}) v^{2}\right] u=0 \\
\Delta^{2} v+\sigma \nabla \cdot\left(|\nabla u|^{p-2} \nabla v\right)+\mu \cdot v+g(\boldsymbol{x}, v)+\left[c(\boldsymbol{x}) u^{2}+d(\boldsymbol{x}) v^{2}\right] v=0
\end{gathered}
$$

where $u=u(\boldsymbol{x}), v=v(\boldsymbol{x}), a(\boldsymbol{x}), b(\boldsymbol{x}), c(\boldsymbol{x})$ and $d(\boldsymbol{x})$ are real-valued functions, $\boldsymbol{x} \in \boldsymbol{R}^{n}, n \geq 2, \alpha, \beta, \sigma, \mu$ are all real constants.

As usual, $\boldsymbol{x}=\left(x_{1}, x_{2}, \cdots, x_{n}\right), \nabla u$ denotes the gradient of $u, \nabla \cdot u$ denotes the divergence of $u$, and $r=|\boldsymbol{x}|$. Also the subscript denotes the partial derivative, thus $u_{s}=\partial u / \partial s$. We also use the notation $u_{r}=\partial u / \partial r=(x / r) \cdot \nabla u$ and $\partial_{j}=\partial / \partial x_{j} \cdot F_{r}(\boldsymbol{x}, s)$ denotes $\partial F(\boldsymbol{x}, s) / \partial r=(\boldsymbol{x} / r) \cdot \nabla_{x} F(\boldsymbol{x}, s) \cdot C^{k}\left(\boldsymbol{R}^{n}\right)$ is the space of functions whose partial derivatives of order up to and including $k$ are continuously differentiable.

Define eight sets of functions $A_{m}\left(\boldsymbol{R}^{n}\right), B_{m}\left(\boldsymbol{R}^{n}\right), D_{h, k}\left(\boldsymbol{R}^{n}\right), E_{m}\left(\boldsymbol{R}^{n}\right), F_{n}\left(\boldsymbol{R}^{n}\right)$, $G_{n}\left(\boldsymbol{R}^{n}\right), N F\left(\boldsymbol{R}^{n}\right)$ and $N G\left(\boldsymbol{R}^{n}\right)$ which we will use in this article:

$$
\begin{aligned}
& A_{m}\left(\boldsymbol{R}^{n}\right)=\left\{a \mid a \in C^{1}\left(\boldsymbol{R}^{n}\right), \sup _{|x| \geq \rho}\left(|x|^{-m}|a(x)|\right)<\infty \text { for some } \rho>0\right. \\
& \text { and } \left.m>0 \text {, and } r a_{r}(\boldsymbol{x})-(n-2) a(\boldsymbol{x}) \leq 0\right\} \\
& B_{m}\left(\boldsymbol{R}^{n}\right)=\left\{b \mid b \in C^{1}\left(\boldsymbol{R}^{n}\right), \sup _{|x| \geq \rho}\left(|\boldsymbol{x}|^{-m}|b(\boldsymbol{x})|\right)<\infty \text { for some } \rho>0\right. \\
& \text { and } \left.m>0 \text {, and } 2 r b_{r}(\boldsymbol{x})-(n-3) b(\boldsymbol{x}) \leq 0\right\} \\
& D_{h, k}\left(\boldsymbol{R}^{n}\right)=\left\{u \mid u \in C^{k}\left(\boldsymbol{R}^{n}\right), \lim _{R \rightarrow \infty}\left(\sup _{|\boldsymbol{x}| \leq R}\left(\left|\boldsymbol{x}^{\alpha}\right|\left|D^{\beta} u(\boldsymbol{x})\right|\right)\right)=0,\right. \\
& \text { for all multi-indices } \boldsymbol{\alpha} \text { and } \boldsymbol{\beta} \in \boldsymbol{N}_{0}^{n} \text { such that }|\boldsymbol{\alpha}| \leq h \text { and } \\
& |\boldsymbol{\beta}| \leq k \text {, where } \boldsymbol{\alpha}=\left(\alpha_{1}, \alpha_{2}, \cdots, \alpha_{n}\right), \boldsymbol{\beta}=\left(\beta_{1}, \beta_{2}, \cdots, \beta_{n}\right) \text {, } \\
& \left.|\alpha|=\alpha_{1}+\alpha_{2}+\cdots+\alpha_{n} \text { and }|\beta|=\beta_{1}+\beta_{2}+\cdots+\beta_{n}\right\} \text {, } \\
& E_{m}\left(\boldsymbol{R}^{n}\right)=\left\{a \mid a \in C^{1}\left(\boldsymbol{R}^{n}\right), \underset{|x| \geq \rho}{\sup }\left(|x|^{-m}|a(\boldsymbol{x})|\right)<\infty \text { for some } \rho>0 \text { and } m>0\right\}, \\
& F_{n}\left(\boldsymbol{R}^{n}\right)=\left\{u \mid \lim _{R \rightarrow \infty}\left(R^{n} \sup _{|x|=R}|F(\boldsymbol{x}, u(\boldsymbol{x}))|\right)=0\right\} \text {, } \\
& G_{n}\left(\boldsymbol{R}^{n}\right)=\left\{v \mid \lim _{R \rightarrow \infty}\left(R^{n} \sup _{|\boldsymbol{x}|=R}|F(\boldsymbol{x}, v(\boldsymbol{x}))|\right)=0\right\} \text {, } \\
& N F\left(\boldsymbol{R}^{n}\right)=\left\{u \mid \int_{\boldsymbol{R}^{n}}\left[n F(\boldsymbol{x}, u(\boldsymbol{x}))+r F_{r}(\boldsymbol{x}, u(\boldsymbol{x}))-((n-1) / 2) f(\boldsymbol{x}, u(\boldsymbol{x})) u(\boldsymbol{x})\right] \mathrm{d} \boldsymbol{x} \leq 0\right\}, \\
& \text { and } \\
& N G\left(\boldsymbol{R}^{n}\right)=\left\{v \mid \int_{\boldsymbol{R}^{n}}\left[n G(\boldsymbol{x}, v(\boldsymbol{x}))+r G_{r}(\boldsymbol{x}, v(\boldsymbol{x}))-((n-1) / 2) g(\boldsymbol{x}, v(\boldsymbol{x})) v(\boldsymbol{x})\right] \mathrm{d} \boldsymbol{x} \leq 0\right\},
\end{aligned}
$$


where $F(x, u)$ and $G(x, v)$ are the antiderivative of $f(x, u)$ with respect to $u$ and $g(x, v)$ with respect to $v$, respectively, such that $F(x, 0)=0$ and $G(x, 0)=0$.

Remark 1. A function $u$ is said to be of decay order $(h, k)$ if and only if $u \in D_{h, k}\left(\boldsymbol{R}^{n}\right)$.

All the functions are assumed to be real-valued.

\section{A Biharmonic Equation with a P-Laplacian Term}

We consider the equation (1.1) in this section. Multiplying both sides of the equation (1.1) by the Morawetz multiplier $\zeta\left(u_{r}+((n-1) u /(2 r))\right)$, where $\zeta \in C^{3}\left(\boldsymbol{R}^{n}\right)$ and $\zeta(\boldsymbol{x})=\zeta(|\boldsymbol{x}|)=\zeta(r)$, we get

$$
\begin{aligned}
0 & =\left[\Delta^{2} u+\alpha \nabla \cdot\left(|\nabla u|^{p-2} \nabla u\right)+\beta \cdot u+f(x, u)\right] \zeta\left(u_{r}+((n-1) u /(2 r))\right) \\
& =\nabla \cdot Y+Z,
\end{aligned}
$$

where $Y$ depends on $\zeta$ and $u$ as well as their partial derivatives up to and including the third order and $F(x, u)$, and

$$
\begin{aligned}
Z= & \left(3 \zeta^{\prime} / 2\right)(\Delta u)^{2}+A\left(u_{r}\right)^{2}+B\left(|\nabla u|^{2}-\left|u_{r}\right|^{2}\right)+C u^{2}+\left(\zeta-r \zeta^{\prime}\right) P \\
& +\alpha\left\{\left((\zeta / r)-\zeta^{\prime}\right)|\nabla u|^{p-2}\left[\left(u_{r}\right)^{2}+((n-1) /(2 r)) u_{r} u\right]\right. \\
& \left.+\left[\left(\zeta^{\prime} / p\right)-(\zeta /(2 p r))((n+1) p-2(n-1))\right]|\nabla u|^{p}\right\} \\
& -\beta\left\{(1 / 2) \zeta_{r}|\nabla u|^{2}+(\nabla u \cdot \nabla \zeta) u_{r}-\zeta_{r}\left|u_{r}\right|^{2}+\left((1 / r) \zeta-\zeta_{r}\right)\left(|\nabla u|^{2}-\left|u_{r}\right|^{2}\right)\right. \\
& \left.+((n-1) /(2 r))\left[(1 / r) \zeta_{r}-(1 / 2)(\Delta \zeta)+\left((n-3) /\left(2 r^{2}\right)\right) \zeta\right] u^{2}\right\} \\
& -\left(\zeta_{r}+((n-1) / r) \zeta\right) F(x, u)+((n-1) /(2 r)) \zeta f(x, u) u-\zeta F_{r}(\boldsymbol{x}, u)
\end{aligned}
$$

where

$$
\begin{gathered}
A=-7 \zeta^{\prime \prime \prime} / 2-(n-1)(n-3)\left(\zeta^{\prime}-(\zeta / r)\right) /\left(2 r^{2}\right), \\
B=-3 \zeta^{\prime \prime \prime} / 2+(n-5) \zeta^{\prime \prime} / r-\left(n^{2}+2 n-19\right)\left(\zeta^{\prime}-\zeta / r\right) /\left(2 r^{2}\right), \\
C=((n-1) / 2)\left[\zeta^{\prime \prime \prime} /(2 r)+(n-3) \zeta^{\prime \prime \prime} /\left(r^{2}\right)+(n-3)(n-7) \zeta^{\prime \prime} /\left(2 r^{3}\right)\right. \\
\left.-3(n-3)(n-5)\left(\zeta^{\prime}-\zeta / r\right) /\left(2 r^{4}\right)\right],
\end{gathered}
$$

and

$$
P=(2 / r)\left[\sum\left(S_{i j} u\right)^{2}-\sum\left(\sum\left(x_{j} / r\right) S_{i j} u\right)^{2}\right] \geq 0,
$$

where

$$
\boldsymbol{S}_{i j} u=\left(x_{i} / r^{3}\right) \sum\left[x_{k}\left(x_{k} \partial_{j}-x_{j} \partial_{k}\right) u_{r}\right]+\partial_{j} \sum\left[\left(x_{k} / r^{2}\right)\left(x_{k} \partial_{i}-x_{i} \partial_{k}\right) u\right] .
$$

Note that we use the Einstein summation notation in the expressions for $P$ and $\boldsymbol{S}_{i j} u$.

Theorem 1

Let $u$ be a $c^{4}$ solution of (1.1) such that $u \otimes D_{n, 3}\left(\boldsymbol{R}^{n}\right) \cap F_{n}\left(\boldsymbol{R}^{n}\right) \cap N F\left(\boldsymbol{R}^{n}\right)$. 
Assume $\beta \leq 0$.

(a) If $p \geq(2 n) /(n+1)$ and $\alpha \leq 0$, then $u \equiv 0$.

(b) If $1<p \leq(2 n) /(n+1)$ and $\alpha \geq 0$, then $u \equiv 0$.

Proof:

Let $R>0$. Integrating both sides of (2.1) in $|x| \leq R$ and using the Divergence theorem, we get

$$
\int_{|x|=R} Y \cdot(x / R) \mathrm{d} s+\int_{|x| \leq R} Z \mathrm{~d} \mathrm{x}=0
$$

Let $R \rightarrow \infty$. We get

$$
\lim _{R \rightarrow \infty} \int_{|x|=R} Y \cdot(x / R) \mathrm{d} s+\int_{R^{n}} Z \mathrm{~d} x=0 .
$$

Thus

$$
\begin{aligned}
0= & \lim _{R \rightarrow \infty} \int_{|x|=R} Y \cdot(\boldsymbol{x} / R) \mathrm{d} s \\
& +\int_{R^{n}}\left\{\left(3 \zeta^{\prime} / 2\right)(\Delta u)^{2}+A\left(u_{r}\right)^{2}+B\left(|\nabla u|^{2}-\left|u_{r}\right|^{2}\right)+C u^{2}+\left(\zeta-r \zeta^{\prime}\right) P\right. \\
& +\alpha\left\{\left((\zeta / r)-\zeta^{\prime}\right)|\nabla u|^{p-2}\left[\left(u_{r}\right)^{2}+((n-1) /(2 r)) u_{r} u\right]\right. \\
& \left.+\left[\left(\zeta^{\prime} / p\right)-(\zeta /(2 p r))((n+1) p-2(n-1))\right]|\nabla u|^{p}\right\} \\
& -\beta\left\{(1 / 2) \zeta_{r}|\nabla u|^{2}+(\nabla u \cdot \nabla \zeta) u_{r}-\zeta_{r}\left|u_{r}\right|^{2}+\left((1 / r) \zeta-\zeta_{r}\right)\left(|\nabla u|^{2}-\left|u_{r}\right|^{2}\right)\right. \\
& \left.+((n-1) /(2 r))\left[(1 / r) \zeta_{r}-(1 / 2)(\Delta \zeta)+\left((n-3) /\left(2 r^{2}\right)\right) \zeta\right] u^{2}\right\} \\
& \left.-\left(\zeta_{r}+((n-1) / r) \zeta\right) F(x, u)+((n-1) /(2 r)) \zeta f(x, u) u-\zeta F_{r}(x, u)\right\} \mathrm{d} \boldsymbol{x},
\end{aligned}
$$

where $A, B, C, P$ are defined as in (2.2)-(2.5).

The above equation (2.6) can be written as

$$
\begin{aligned}
0= & \lim _{R \rightarrow \infty} \int_{|x|=R} Y \cdot(x / R) \mathrm{d} s \\
& +\int_{R^{n}}\left\{\left(3 \zeta^{\prime} / 2\right)(\Delta u)^{2}+A\left(u_{r}\right)^{2}+B\left(|\nabla u|^{2}-\left|u_{r}\right|^{2}\right)+C u^{2}+\left(\zeta-r \zeta^{\prime}\right) P\right. \\
& +\alpha\left\{\left((\zeta / r)-\zeta^{\prime}\right)|\nabla u|^{p-2}\left[\left(u_{r}\right)^{2}+((n-1) /(2 r)) u_{r} u\right]\right. \\
& \left.+\left[\left(\zeta^{\prime} / p\right)-(\zeta /(2 p r))((n+1) p-2(n-1))\right]|\nabla u|^{p}\right\} \\
& -\beta\left\{(1 / 2) \zeta_{r}|\nabla u|^{2}+(\nabla u \cdot \nabla \zeta) u_{r}-\zeta_{r}\left|u_{r}\right|^{2}+\left((1 / r) \zeta-\zeta_{r}\right)\left(|\nabla u|^{2}-\left|u_{r}\right|^{2}\right)\right. \\
& \left.+((n-1) /(2 r))\left[(1 / r) \zeta_{r}-(1 / 2)(\Delta \zeta)+\left((n-3) /\left(2 r^{2}\right)\right) \zeta\right] u^{2}\right\} \mathrm{d} \boldsymbol{x} \\
= & \left.\int_{\boldsymbol{R}^{n}}\left(\zeta_{r}+((n-1) / r) \zeta\right) F(\boldsymbol{x}, u)-((n-1) /(2 r)) \zeta f(\boldsymbol{x}, u) u+\zeta F_{r}(\boldsymbol{x}, u)\right\} \mathrm{d} \boldsymbol{x},
\end{aligned}
$$

Let $\zeta(\boldsymbol{x})=\zeta(|\boldsymbol{x}|)=\zeta(r)=r$.

Since $u$ is assumed to be of decay order $(n, 3)$ and $u \in F_{n}\left(\boldsymbol{R}^{n}\right)$, $\lim _{R \rightarrow \infty} \int_{|x|=R} Y \cdot(x / R) \mathrm{d} \boldsymbol{s}=0$, after substituting $\zeta$ by $r$.

\section{Thus}




$$
\begin{aligned}
& \int_{\boldsymbol{R}^{n}}\left\{(3 / 2)(\Delta u)^{2}+\alpha[(1 / p)-(1 /(2 p))((n+1) p-2(n-1))]|\nabla u|^{p}-(\beta / 2)|\nabla u|^{2}\right\} \mathrm{d} \boldsymbol{x} \\
& =\int_{\boldsymbol{R}^{n}}\left[n F(\boldsymbol{x}, u)-((n-1) / 2) f(\boldsymbol{x}, u) u+r F_{r}(\boldsymbol{x}, u)\right] \mathrm{d} \boldsymbol{x} .
\end{aligned}
$$

To prove the assertion (a), since $\beta \leq 0$, we have

$$
\begin{aligned}
& \int_{\boldsymbol{R}^{n}}\left\{(3 / 2)(\Delta u)^{2}+\alpha[(1 / p)-(1 /(2 p))((n+1) p-2(n-1))]|\nabla u|^{p}\right\} \mathrm{d} \boldsymbol{x} \\
& \leq \int_{\boldsymbol{R}^{n}}\left[n F(\boldsymbol{x}, u)-((n-1) / 2) f(\boldsymbol{x}, u) u+r F_{r}(\boldsymbol{x}, u)\right] \mathrm{d} \boldsymbol{x}
\end{aligned}
$$

Thus

$$
\begin{aligned}
& \int_{\boldsymbol{R}^{n}}\left\{(3 / 2)(\Delta u)^{2}+\alpha[(2 n-(n+1) p) /(2 p)]|\nabla u|^{p}\right\} \mathrm{d} \boldsymbol{x} \\
& \leq \int_{\boldsymbol{R}^{n}}\left[n F(\boldsymbol{x}, u)-((n-1) / 2) f(\boldsymbol{x}, u) u+r F_{r}(\boldsymbol{x}, u)\right] \mathrm{d} \boldsymbol{x}
\end{aligned}
$$

Since $\alpha \leq 0, p \geq(2 n) /(n+1)$, and $u \in N F\left(\boldsymbol{R}^{n}\right)$, we have

$$
0 \leq \int_{\boldsymbol{R}^{n}}(3 / 2)(\Delta u)^{2} \mathrm{~d} \boldsymbol{x} \leq \int_{\boldsymbol{R}^{n}}\left[n F(\boldsymbol{x}, u)-((n-1) / 2) f(\boldsymbol{x}, u) u+r F_{r}(\boldsymbol{x}, u)\right] \mathrm{d} \boldsymbol{x} \leq 0,
$$

Thus $\int(3 / 2)(\Delta u)^{2} \mathrm{~d} \boldsymbol{x}=0$. Since $u \in D_{n, 3}\left(R_{n}\right), u \equiv 0$

Assertib ${ }^{n}$ (b) follows with a similar argument from (2.7).

Remark 2. As an example for $f(x, u)$, let $f(x, u)=q(x)|u|^{s-1} u, s \geq 1$. Then $F(\boldsymbol{x}, u)=(1 /(s+1)) q(x)|u|^{s+1}$, where $q(\boldsymbol{x})=q(|\boldsymbol{x}|)=q(r)$.

Assume $q \in E_{m}\left(\boldsymbol{R}^{n}\right)$.

For $u$ to be in $F_{n}\left(\boldsymbol{R}^{n}\right)$, we need

$$
\lim _{R \rightarrow \infty}\left(R^{n} \sup _{|x|=R}|F(x, u(x))|\right)=0,
$$

that is,

$$
\lim _{R \rightarrow \infty}\left(R^{n} \sup _{|x|=R}\left(q(|x|)|u(x)|^{s+1}\right)\right)=0,
$$

This would be satisfied if

$$
\lim _{R \rightarrow \infty}\left(R^{n} \sup _{|x|=R}\left(|\boldsymbol{x}|^{m}|u(\boldsymbol{x})|^{s+1}\right)\right)=0 .
$$

The above condition (2.8) would be satisfied if $u$ is of decay order $(n+m, 0)$. As for $u$ to be in $N F\left(\boldsymbol{R}^{n}\right)$, since

$$
\begin{aligned}
& n F(\boldsymbol{x}, u)+r F_{r}(\boldsymbol{x}, u)-((n-1) / 2) f(\boldsymbol{x}, u) u \\
& =\left[(n /(s+1)) q+(r /(s+1)) q_{r}-((n-1) / 2) q\right]|u|^{s+1},
\end{aligned}
$$

$u$ would be in $N F\left(\boldsymbol{R}^{n}\right)$ if $(n /(s+1)) q+(r /(s+1)) q_{r}-((n-1) / 2) q \leq 0$.

Thus, if $r q_{r} \leq((n s-n-s-1) / 2) q$, then $u$ is in $N F\left(\boldsymbol{R}^{n}\right)$.

Therefore, if $u$ is of decay order $(n+m, 1)$ and $r q_{r} \leq((n s-n-s-1) / 2) q, u$ satisfies the assumptions of Theorem 1 on $u$.

Remark 3. A similar conclusion can be obtained for $f(x, u)=q_{1}(x)|u|^{a-1} u+q_{2}(x)|u|^{b-1} u$, where $a>b \geq 1$. 


\section{A System of Biharmonic Equations with p-Laplacian Terms}

We consider the system (1.4.a) and (1.4.b) in this section. Let $F(x, u)$ and $G(x, v)$ be the antiderivatives of $f(x, u)$ with respect to $u$ and $g(x, v)$ with respect to $V$, respectively, such that $F(x, 0)=0$ and $G(x, 0)=0$.

Assume also $b(\boldsymbol{x})=c(\boldsymbol{x})$. Multiplying both sides of (1.4.a) by $\zeta\left(u_{r}+((n-1) u /(2 r))\right)$ and both sides of (1.4.b) by $\zeta\left(v_{r}+((n-1) v /(2 r))\right)$, then adding them up, we get

$$
\begin{aligned}
0= & \left(\Delta^{2} u+\alpha \nabla \cdot\left(|\nabla u|^{p-2} \nabla u\right)+\beta \cdot u+f(\boldsymbol{x}, u)+\left[a(\boldsymbol{x}) u^{2}+b(\boldsymbol{x}) v^{2}\right] u\right) \zeta\left(u_{r}+((n-1) u /(2 r))\right) \\
& +\left(\Delta^{2} v+\sigma \nabla \cdot\left(|\nabla v|^{p-2} \nabla v\right)+\mu \cdot v+g(\boldsymbol{x}, v)+\left[c(\boldsymbol{x}) u^{2}+d(\boldsymbol{x}) v^{2}\right] v\right) \zeta\left(v_{r}+((n-1) v /(2 r))\right) \\
= & \nabla \cdot Y+Z,
\end{aligned}
$$

where $Y$ depends on $\zeta, u$, and $v$ as well as their partial derivatives up to and including the third order, $F(x, u), G(x, v), \alpha, \beta, \sigma, \mu, a(x), b(x), c(x)$, and $d(\boldsymbol{x})$. Here we assume $b(\boldsymbol{x})=c(\boldsymbol{x}) . Z$ is similar to Section 2 with appropriate modification to allow terms containing $v, \sigma, \mu, a(x), b(x), c(x)$, and $d(\boldsymbol{x})$.

\section{Theorem 2}

Let $\mathrm{u}$ and $\mathrm{v}$ be $C^{4}$ solutions of the system (1.4.a) and (1.4.b) with $b(\boldsymbol{x})=c(\boldsymbol{x})$. Assume $a \in A_{k, 2}\left(\boldsymbol{R}^{n}\right), b \in B_{m, 2}\left(\boldsymbol{R}^{n}\right)$ and $d \in A_{h, 2}\left(\boldsymbol{R}^{n}\right)$. Let $s=\max \{k, m, h, n\}$. Assume $\beta \leq 0$ and $\mu \leq 0$.

Assume further that $u \in D_{s, 3}\left(\boldsymbol{R}^{n}\right) \cap F_{n}\left(\boldsymbol{R}^{n}\right) \cap N F\left(\boldsymbol{R}^{n}\right)$ and $v \in D_{s, 3}\left(\boldsymbol{R}^{n}\right) \cap G_{n}\left(\boldsymbol{R}^{n}\right) \cap N G\left(\boldsymbol{R}^{n}\right)$.

(a) If $p \geq(2 n) /(n+1), \alpha \leq 0$ and $\sigma \leq 0$, then $u \equiv 0$ and $v \equiv 0$.

(b) If $1<p \leq(2 n) /(n+1), \alpha \geq 0$, and $\sigma \geq 0$, then $u \equiv 0$ and $v \equiv 0$.

Proof:

Let $\zeta(\boldsymbol{x})=\zeta(|\boldsymbol{x}|)=\zeta(r)=r$. Following the same steps as in Theorem 1, we get

$$
\begin{aligned}
0 \leq & \int_{\boldsymbol{R}^{n}}(3 / 2)\left((\Delta u)^{2}+(\Delta v)^{2}\right) \mathrm{d} \boldsymbol{x} \\
\leq & \int_{\boldsymbol{R}^{n}}\left\{\left[n F(\boldsymbol{x}, u)-((n-1) / 2) f(\boldsymbol{x}, u) u+r F_{r}(\boldsymbol{x}, u)\right]\right. \\
& +\left[n G(\boldsymbol{x}, v)-((n-1) / 2) g(\boldsymbol{x}, v) v+r G_{r}(\boldsymbol{x}, v)\right] \\
& +(1 / 4)\left[r a_{r}(\boldsymbol{x})-(n-2) a(\boldsymbol{x})\right] u^{4} \\
& +(1 / 4)\left[r d_{r}(\boldsymbol{x})-(n-2) d(\boldsymbol{x})\right] v^{4} \\
& \left.+(1 / 2)\left[r b_{r}(\boldsymbol{x})-((n-3) / 2) b(\boldsymbol{x})\right] u^{2} v^{2}\right\} \mathrm{d} \boldsymbol{x} \\
\leq & 0,
\end{aligned}
$$

since $a \in A_{k}\left(\boldsymbol{R}^{n}\right), b \in B_{m}\left(\boldsymbol{R}^{n}\right), d \in A_{h}\left(\boldsymbol{R}^{n}\right), u \in D_{s, 3}\left(\boldsymbol{R}^{n}\right) \cap F_{n}\left(\boldsymbol{R}^{n}\right) \cap N F\left(\boldsymbol{R}^{n}\right)$ and $v \in D_{s, 3}\left(\boldsymbol{R}^{n}\right) \cap G_{n}\left(\boldsymbol{R}^{n}\right) \cap N G\left(\boldsymbol{R}^{n}\right)$.

Thus

$$
\int_{\boldsymbol{R}^{n}}(3 / 2)\left((\Delta u)^{2}+(\Delta v)^{2}\right) \mathrm{d} \boldsymbol{x}=0 .
$$


Since $u$ and $v \in D_{s, 3}\left(\boldsymbol{R}^{n}\right), u \equiv 0$ and $v \equiv 0$.

\section{Acknowledgements}

A partial result of this work was presented in the 2016 International Workshop on Geometric Analysis \& Subelliptic PDEs, May 24-26, 2016, Taipei, and NCTS International Workshop on Harmonic Analysis and Geometric Analysis, May 23-25, 2017, Taipei. The author wishes to thank the meeting organizer, Dr. DerChen Chang, for the hospitality and the support during the workshop.

\section{References}

[1] Strauss, W.A. (1977) Existence of Solitary Waves in High Dimensions. Communications in Mathematical Physics, 55, 149-162. https://doi.org/10.1007/BF01626517

[2] Abrahams, I.D. and Davis, A.M.J. (2002) Deflection of a Partially Clamped Elastic Plate, IUTAM Symposium on Diffraction and Scattering in Fluid Mechanics and Elasticity. Fluid Mechanics and Its Application, 68, 303-312. https://doi.org/10.1007/978-94-017-0087-0_33

[3] Alves, C.O., do Ó, J.M. and Miyagaki, O.H. (2003) On a Class of Singular Biharmonic Problems Involving Critical Exponents. Journal of Mathematical Analysis and Applications, 277, 12-26. https://doi.org/10.1016/S0022-247X(02)00283-4

[4] An, Y. and Liu, R. (2008) Existence of Nontrivial Solutions of an Asymptotically Linear Fourth-Order Elliptic Equation. Nonlinear Analysis, 68, 3325-3331. https://doi.org/10.1016/j.na.2007.03.028

[5] Ang, D.D. and Dimh, A.P.N. (1988) Strong Solutions of a Quasilinear Wave Equation with Nonlinear Damping. SIAM Journal on Mathematical Analysis, 19, 337 347. https://doi.org/10.1137/0519024

[6] Barenblatt, G.I. and Vazquez, J.L. (2004) Nonlinear Diffusion and Image Contour Enhancement. Interfaces Free Bound, 6, 31-54. https://doi.org/10.4171/IFB/90

[7] Barrett, J.W. and Liu, W. (1994) Finite Element Approximation of the Parabolic p-Laplacian. SIAM Journal on Numerical Analysis, 31, 413-428. https://doi.org/10.1137/0731022

[8] Bermejo, R. and Infante, J.A. (2000) A Multigrid Algorithm for the p-Laplacian. SIAM Journal on Scientific Computing, 21, 1774-1789. https://doi.org/10.1137/S1064827598339098

[9] Biazutti, A.C. (1995) On a Nonlinear Evolution Equation and Its Applications. Nonlinear Analysis, 24, 1221-1234.

[10] Caffarelli, L.A. and Wolanski, N. (1990) $C^{1, \alpha}$-Regularity of the Free Boundary for the N-Dimensional Porous Media Equation. Communications on Pure and Applied Mathematics, 43, 885-902. https://doi.org/10.1002/cpa.3160430704

[11] Carrião, P.C., Demarque, R. and Miyagaki, O.H. (2014) Nonlinear Biharmonic Problems with Singular Potentials. Communications on Pure and Applied Analysis, 13, 2141-2154. https://doi.org/10.3934/cpaa.2014.13.2141

[12] Catté, F., Lions, P.-L., Morel, J.-M. and Coll, T. (1992) Image Selective Smoothing and Edge Detection by Nonlinear Diffusion. SIAM Journal on Numerical Analysis, 29, 182-193. https://doi.org/10.1137/0729012

[13] Feng, X. and Neilan, M. (2009) Vanishing Moment Method and Moment Solution for Second Order Fully Nonlinear Partial Differential Equations. Journal of Scientific Computing, 38, 74-98. https://doi.org/10.1007/s10915-008-9221-9 
[14] Gazzola, F. and Grunau, H.C. (2006) Radial Entire Solutions for Supercritical Biharmonic Equations. Mathematische Annalen, 334, 905-936. https://doi.org/10.1007/s00208-005-0748-x

[15] Gil, O. and Vázquez, J.L. (1997) Focusing Solutions for the p-Laplacian Evolution Equation. Advances in Differential Equations, 2, 183-202.

[16] Harrabi, A. (2014) Fourth-Order Elliptic Equations. Advanced Nonlinear Studies, 14, 593-604. https://doi.org/10.1515/ans-2014-0304

[17] $\mathrm{Hu}, \mathrm{S}$. and Wang, L. (2014) Existence of Nontrivial Solutions for Fourth-Order Asymptotically Linear Elliptic Equations. Nonlinear Analysis, 94, 120-132.

[18] Huang, Y., Li, R. and Liu, W. (2007) Preconditioned Descent Algorithms for pLaplacian. Journal of Scientific Computing, 32, 343-371. https://doi.org/10.1007/s10915-007-9134-Z

[19] Kamin, S. and Vázquez, J.L. (1988) Fundamental Solutions and Asymptotic Behaviour for the p-Laplacian Equation. Revista Matemática Iberoamericana, 4, 339-354. https://doi.org/10.4171/RMI/77

[20] Lazer, A.C. and McKenna, P.J. (1990) Large-Amplitude Periodic Oscillations in Suspension Bridge: Some New Connections with Nonlinear Analysis. SIAM Review, 32, 537-578. https://doi.org/10.1137/1032120

[21] Lazer, A.C. and McKenna, P.J. (1994) Global Bifurcation and a Theorem of Tarantello. Journal of Mathematical Analysis and Applications, 181, 648-655.

[22] Lee, K., Petrosyan, A. and Vázquez, J.L. (2006) Large-Time Geometric Properties of Solutions of the Evolutions p-Laplacian Equation. Journal of Differential Equations, 229, 389-411.

[23] Lee, K. and Vázquez, J.L. (2003) Geometrical Properties of Solutions of the Porous Medium Equation for Large Times. Indiana University Mathematics Journal, 52, 991-1016. https://doi.org/10.1512/iumj.2003.52.2200

[24] Li, H.-L. and Zhao, J.-N. (2000) Regularity of Solutions for the Evolution p-Laplacian Equations. Northeastern Mathematical Journal, 16, 96-98.

[25] Li, T., Sun, J. and Wu, T.F. (2015) Existence of Homoclinic Solutions for a Fourth Order Differential Equation with a Parameter. Applied Mathematics and Computation, 251, 499-506.

[26] Liu, J., Chen, S. and Wu, X. (2012) Existence and Multiplicity of Solutions for a Class of Fourth-Order Elliptic Equations in $R^{n}$. Journal of Mathematical Analysis and Applications, 395, 608-615.

[27] Liu, W. and Yan, N. (2001) Quasi-Norm Local Error Estimators for p-Laplacian. SIAM Journal on Numerical Analysis, 39, 100-127. https://doi.org/10.1137/S0036142999351613

[28] Micheletti, A.M. and Pistoia, A. (1998) Nontrivial Solutions for Some Fourth Order Semilinear Elliptic Problems. Nonlinear Analysis, 34, 509-523.

[29] Micheletti, A.M. and Pistoia, A. (1998) Multiplicity Results for a Fourth Order Semilinear Elliptic Problem. Nonlinear Analysis, 31, 895-908.

[30] Nakao, M. (1978) A Difference Inequality and Its Applications to Nonlinear Evolution Equations. Journal of the Mathematical Society of Japan, 30, 747-762. https://doi.org/10.2969/jmsj/03040747

[31] Nakao, M. (1992) Energy Decay for the Quasilinear Wave Equation with Viscosity. Mathematische Zeitschrift, 219, 289-299. https://doi.org/10.1007/BF02572366

[32] Perona, P. and Malik, J. (1990) Scale Space and Edge Detection Using Anisotropic Diffusion. IEEE Transactions on Pattern Analysis and Machine Intelligence, 12, 
629-639. https://doi.org/10.1109/34.56205

[33] Raviart, P.A. (1970) Sur la résolution de certaines équations paraboliques non linéaires. Journal of Functional Analysis, 5, 299-328.

[34] Sakaguchi, S. (1997) Spatial Critical Points of Nonnegative Solutions of the Evolution p-Laplacian Equation: The Fast Diffusion Case. Differential and Integral Equations, 10, 1049-1063.

[35] Sun, J. and Wu, T.F. (2014) Two Homoclinic Solutions for a Nonperiodic Fourth Order Differential Equation with a Perturbation. Journal of Mathematical Analysis and Applications, 413, 622-632.

[36] Wang, J. and Zhang, Y. (2012) A Biharmonic Eigenvalue Problem and Its Application. Acta Mathematica Scientia, 32, 1213-1225.

[37] Webb, G.F. (1980) Existence and Asymptotic for a Strongly Damped Nonlinear Wave Equation. Canadian Journal of Mathematics, 32, 631-643. https://doi.org/10.4153/CJM-1980-049-5

[38] Ye, Y. and Tang, C. (2012) Infinitely Many Solutions for Fourth-Order Elliptic Equations. Journal of Mathematical Analysis and Applications, 394, 841-854.

[39] Ye, Y. and Tang, C. (2013) Existence and Multiplicity of Solutions for Fourth-Order Elliptic Equations in $R^{n}$. Journal of Mathematical Analysis and Applications, 406, 335-351.

[40] Yin, Y. and Wu, X. (2011) High Energy Solutions and Nontrivial Solutions for Fourth-Order Elliptic Equations. Journal of Mathematical Analysis and Applications, 375, 699-705.

[41] Zhou, G. and Feng, C. (2013) The Steepest Descent Algorithm without Line Search for p-Laplacian. Applied Mathematics and Computation, 224, 36-45.

[42] Zhou, G., Huang, Y. and Feng, C. (2005) Preconditioned Hybrid Conjugate Gradient Algorithm for p-Laplacian. International Journal of Numerical Analysis and Modeling, 2, 123-130.

[43] Levandosky, S.P. and Strauss, W.A. (2000) Time Decay for the Nonlinear Beam Equations. Methods and Applications of Analysis, 7, 479-488.

[44] Morawetz, C.S. (1968) Time Decay for Nonlinear Klein-Gordon Equation. Proceedings of the Royal Society A, 306, 291-296.

https://doi.org/10.1098/rspa.1968.0151

[45] Strauss, W.A. (1990) Nonlinear Wave Equations. CBMS Regional Conference Series in Mathematics, No. 73, American Mathematical Society, Providence. 
Submit or recommend next manuscript to SCIRP and we will provide best service for you:

Accepting pre-submission inquiries through Email, Facebook, LinkedIn, Twitter, etc. A wide selection of journals (inclusive of 9 subjects, more than 200 journals)

Providing 24-hour high-quality service

User-friendly online submission system

Fair and swift peer-review system

Efficient typesetting and proofreading procedure

Display of the result of downloads and visits, as well as the number of cited articles Maximum dissemination of your research work

Submit your manuscript at: http://papersubmission.scirp.org/

Or contact am@scirp.org 